\title{
Advances in Clinical Diagnosis and Management of Chikungunya Virus Infection
}

\author{
Claudio Soto-Garita, MSc Candidate ${ }^{1}$ \\ Jean-Paul Carrera, MSC ${ }^{2,3}$ \\ Sandra López-Vergès, $P h D^{2}$ \\ Eugenia Corrales-Aguilar, $P h D^{1, *}$
}

\author{
Address \\ ${ }^{*}, 1$ Virology - Research Center for Tropical Diseases (CIET), Faculty of Microbiology, \\ University of Costa Rica, San José, Costa Rica \\ Email: eugenia.corrales@ucr.ac.cr \\ ${ }^{2}$ Department of Research in Virology, Gorgas Memorial Institute of Health Studies, \\ Panama City, Panama \\ ${ }^{3}$ Deparment of Preclinical Medicine, Columbus University, Panama City, Panama
}

Published online: 8 May 2018

(C) Springer Science+Business Media, LLC, part of Springer Nature 2018

This article is part of the Topical Collection on Neglected Tropical Diseases

Keywords Chikungunya virus - Chikungunya diagnosis - Chikungunya clinical management - Arbovirus differential diagnosis

\begin{abstract}
Purpose of review In this review, we attempt to give the reader an update on clinical diagnosis, management, and treatment of Chikungunya virus (CHIKV) infection regarding the course of the disease and the role of clinical laboratory. CHIKV infection evolves through three phases: acute, sub-acute, and chronic. Protocol for patient assessment and management should be in agreement with each phase requirements.

Recent findings Despite its benignity, CHIKV infection has shown an increase in severe cases during recent outbreaks. Among the most affected group are neonates, elderly population, and patients with co-morbidities. Furthermore, since CHIKV geographical distribution and clinical spectrum overlaps with other arboviruses, differential diagnosis becomes crucial for physicians to ensure good management and treatment for patients. In this review, we describe and compare the main symptoms, complications, and risk groups for CHIKV, Dengue (DENV), and Zika (ZIKV) infections, regarding key features will guide to an accurate diagnosis. Summary CHIKV is an emergent and reemergent arbovirus that arrived in the Americas in 2014, causing thousands of infections. The acute febrile phase is similar to that of dengue but with a higher proportion of debilitating arthralgia that can persist for months or even years after infection. Severe forms of CHIKV
\end{abstract}


infection can lead to chronic polyarthralgia, neurologic symptoms, or multiorgan failure. Treatment of CHIKV infection is mainly focused on supportive care, which includes the use of analgesic and anti-inflammatory medication, rehydration, and rest.

\section{Introduction}

\section{Chikungunya virus}

Chikungunya is one of the most important emergent and reemergent arboviral disease in tropical and subtropical regions and it is transmitted by Aedes mosquitoes [1]. Chikungunya virus (CHIKV) was first isolated and described during a febrile illness outbreak in the Makonde Plateau, a southern province of Tanzania, between 1952 and 1953 [2]. Its name is derived from the Swahili or Makonde expression "Kun qunwala," which means "to become contorted" or "that which bends up," referring to the disabling arthritic pain experienced during the disease [3].

CHIKV belongs to the Alphavirus genus within the Togaviridae family; specifically to the Semliki Forest antigenic complex along with other arboviruses such as Mayaro and O'nyong-nyong [4]. CHIKV has a positivesense single-stranded RNA genome with two open reading frames (ORFs): the $5^{\prime}$ ORF code for non-structural proteins (NSP1, 2, 3, and 4) and the 3' ORF code for a polyprotein containing the structural proteins (capsid and envelope proteins). The viral particle possesses a spherical structure with approximately $65 \mathrm{~nm}$ in diameter; it is composed by 240 copies of the capsid protein and surrounded by a lipid envelope, which has trimershaped spikes of envelope proteins [5].

Phylogenetic and evolutionary analyses have revealed three main genotypes for CHIKV: the Asian, the West African and the East-Central-South African (ECSA). Each genotype subdivides in several lineages, which differ in geographic distribution, antigenic properties, and viral fitness in vertebrate and invertebrate hosts [6].

\section{Re-emergence and expansion of CHIKV}

Two transmission cycles have been described for CHIKV. The enzootic or sylvatic cycle is found mainly in the African continent and involves non-human primates and sylvatic Aedes mosquitoes as main vectors. Spillover events from this cycle have been followed by the initiation of an urban transmission cycle, involving humans as the vertebrate hosts and Aedes aegypti and Ae. albopictus as vectors [7•].

Since its first description in the 1950s, and supported by retrospective analysis, several authors have suggested that during previous chikungunya outbreaks, clinical cases have been masked and incorrectly classified as Dengue virus (DENV) infections, given the overlapping clinical presentation $[8,9]$.

Between the 1950s and 2000s, the disease was exclusively reported in the African and Asian continents and marked by infrequent outbreaks and periods of inactivity. But since the year 2000, larger and more severe recurring outbreaks have been documented in other endemic and naïve regions [10]. During the epidemic wave between 2000 and 2007, there were large outbreaks in costal Kenya that spread to the La Réunion, India, Mauritius, Gabon, Thailand, and the Republic of Congo [11-13]. A new strain classified as Indian Ocean Lineage (IOL) related to the ECSA genotype arose from this re-emergence $[14 \bullet \bullet]$. Point mutations in the envelope protein of the IOL strain have been associated with a better adaptation to Ae. albopictus, thus contributing to the size of the epidemic [15]. In 2007, local transmission of CHIKV was reported in northern Italy, where Ae. albopictus was found as the main vector, raising concerns of a potential introduction of CHIKV into the European and then the American continents [16]. Despite the amount of viremic travelers entering the Americas during this epidemic wave, there were no locally acquired infections reported [17]. It was until 2013 when the first CHIKV urban cycle was described in the Caribbean island of St. Martin with virus isolates identified as the Asian genotype [18]. This event marked the establishment of CHIKV in the Americas, now endemic in almost all countries of Latin America. Furthermore, the cocirculation of both the Asian and ECSA genotypes in Brazil since 2014 raises the risk of spreading of the former in Americas and beyond [19, 20]. 


\section{CHIKV disease and criteria for diagnosis}

CHIKV infection causes an acute febrile disease, similar to dengue and other arboviruses infections, typically accompanied by disabling polyarthralgia that can persist for weeks, months, or years [21]. In addition to fever and arthralgia, symptoms such as asthenia, myalgia, headache, maculopapular rash, and nausea are commonly observed in CHIKV patients. CHIKV infection is diagnosed on the basis of clinical, epidemiological, and laboratory criteria. Due to the overlapping geographic distribution and symptoms between CHIKV and other arboviruses such as DENV and Zika virus (ZIKV), accurate diagnosis and clinical management is challenging [22]. In this review, we attempt to address the new advances in each of these criteria, along with the clinical management and reported treatment of the chikungunya infection.

\section{Clinical diagnosis, management and treatment of CHIKV infection}

\section{Clinical spectrum of CHIKV infection}

Clinical manifestations caused by CHIKV infection range from asymptomatic or subclinical infection and undifferentiated fever with arthralgia to more severe forms, such as chronic polyarthralgia, encephalitis, and/or multiorgan failure. The percentage of subclinical infections fluctuates between epidemics and it depends on factors such as age, naïve population/herd immunity, and the circulating strain [6]. Typically, asymptomatic infections in CHIKV outbreaks are rare. Seroepidemiological studies revealed a 3 to $25 \%$ of subclinical presentation in the La Réunion and Mayotte outbreaks [13, 23, 24]. Nevertheless, studies conducted in recent outbreaks in the Nicaragua and Philippines, where the Asian genotype was the circulating strain, showed a 58 to $80 \%$ unapparent CHIKV infection, respectively $[25,26]$.

The symptomatic course of the disease progresses through three phases: acute, post-acute, and chronic [27]. The incubation period after the mosquito bite ranges from 1 to 12 days, with an average of 2 to 4 days. (Table 1) [28]. The acute phase occurs with a sudden fever onset, described as high degree (above $39{ }^{\circ} \mathrm{C}$ ), accompanied by joint pain in almost all patients. Symmetrical polyarthralgia with swelling and other signs of inflammation in more than one joint is a common feature. Wrists, ankles, fingers, elbows, toes, and knees are the most recurrently affected. The joint pain and stiffness is reported to be worse during the morning, particularly after awakening, and relieved by mild exercise but more severe after intense activity. Patients with underlying rheumatic conditions, such as osteoarthritis, are particularly susceptible. Additionally, patients have reported intense myalgia, headache, photophobia, fatigue, nausea, vomiting, diarrhea, adenopathy, and conjunctivitis [29-31].

The cutaneous manifestations of CHIKV infection are observed in up to $80 \%$ of patients between the second and the fifth day after the onset of fever and arthralgia [27]. Transient skin rash, with or without pruritus, presented as a maculopapular exanthema involving primarily face, torso, and extremities is the most common manifestation [32]. Pre-existing dermatoses, such as psoriasis, can be exacerbated. Pediatric patients can show vesicular exanthema and bullous lesions with petechiae [33].

Despite the low mortality of CHIKV infection (less than one per 1000 individuals), severity augments in some age groups (elders and infants) and patients with certain underlying chronic diseases [34]. Heart and multiorgan failure, hepatitis, and encephalitis are among most common severe 
Table 1. Comparison of main clinical and laboratory findings in CHIKV, DENV, and ZIKV infection

\begin{tabular}{|c|c|c|c|}
\hline $\begin{array}{l}\text { Clinical or laboratory } \\
\text { findings }\end{array}$ & Chikungunya (CHIKV) & Dengue (DENV) & Zika (ZIKV) \\
\hline Fever (above $39^{\circ} \mathrm{C}$ ) & +++ & ++ & + \\
\hline Myalgia & ++ & ++ & + \\
\hline Arthralgia & +++ & + & + \\
\hline Headache & ++ & ++ & + \\
\hline Rash (exanthema) & ++ & ++ & ++ \\
\hline Retro-orbital pain & $+/-$ & ++ & + \\
\hline Conjunctivitis & $+/-$ & - & ++ \\
\hline Hypotension & $+/-$ & ++ & - \\
\hline Bleeding & $+/-$ & ++ & - \\
\hline Neutropenia & + & +++ & - \\
\hline Thrombocytopenia & + & +++ & - \\
\hline Increased hematocrit & - & ++ & - \\
\hline Main complications & $\begin{array}{l}\text { - Encephalitis } \\
\text { - Multi-organic failure } \\
\text { - Hepatitis }\end{array}$ & $\begin{array}{l}\text { - Shock syndrome } \\
\text { - Hemorrhage } \\
\text { - Hepatitis }\end{array}$ & $\begin{array}{l}\text { - Guillian-Barré syndrome } \\
\text { - Congenital microcephaly }\end{array}$ \\
\hline Main long-term sequels & $\begin{array}{l}\text { - Chronic inflammatory } \\
\text { rheumatism and arthritis }\end{array}$ & - None & $\begin{array}{c}\text { - Neurocognitive development } \\
\text { delay due to microcephaly }\end{array}$ \\
\hline High-risk population & $\begin{array}{l}\text { - Elders } \\
\text { - Infants } \\
\text { - Chronically ill patients }\end{array}$ & $\begin{array}{l}\text { - Patients with prior } \\
\text { infection with DENV }\end{array}$ & - Pregnant women \\
\hline Incubation period & $3-7$ days & $3-10$ days & $3-12$ days \\
\hline Symptoms duration & $\begin{array}{l}2-10 \text { days (acute phase) } \\
\text { Arthralgia can persist for months } \\
\text { or even years }\end{array}$ & $2-10$ days & 4-7 days \\
\hline
\end{tabular}

presentations of the disease and causes of death [35]. In pediatric population, dermatological and neurological complications are more frequent and polyarthralgia is less recurrent [33]. Newborns can acquire CHIKV via perinatal transmission and can present encephalopathy with persistent disabilities [36]. Co-morbidities such as diabetes, cardiovascular, respiratory, renal, and autoimmune diseases increase the risk of developing severe CHIKV infection, especially in elderly patients [37].

CHIKV infection courses as a self-limiting illness in most patients, with mild to advanced improvement reported within the first and second weeks after the acute phase. An important number of patients do not completely recover after this period and enter a post-acute phase, which comprises the first 3 months after the acute phase $[13,24]$. During this period, patients exhibit slow clinical improvement and complete resolution of arthralgia may not be successful. The joint pain may persist as uninterrupted or as intermittent polyarthralgia aggravated by the cold or upon awakening [27].

If the aforementioned symptoms persist after 3 months, the patient enters to the chronic phase. Between 40 and $80 \%$ of symptomatic patients advance to this stage and have three possible outcomes: (a) complete clinical improvement 
(spontaneously or after treatment) without sequels, (b) prolonged persistence of the polyarthralgia, and (c) the aggravation of the disease due to continuous inflammatory reaction $[23,38]$. The majority of patients fit in the first case scenario and recover in weeks or even months. Approximately $10 \%$ of the symptomatic patients report persistence of joint involvement 3 years after CHIKV infection [39]. The arthralgia is present in the same joints involved during the acute phase and is generally less severe [38]. Several risk factors have been studied for the persistence of the arthralgia in the chronic phase [40]. Increased age (above 45 years old), underlying joint involvement, higher viral load, and intensity of symptoms during the acute phase are high risk factors for developing persistent illness [40].

Despite its benignity, CHIKV infection could lead to severe disabling pain during the acute, sub-acute, and chronic phases. Inability in performing daily activities as a result of CHIKV disease could lead to sadness and depression, as reported by several patients [39]. Fluctuations in disease severity and mortality rates during outbreaks and the reduction in patients' quality of life should be taken into consideration in the understanding and management of CHIKV infection among healthcare professionals.

\section{Case definition of CHIKV disease and differential diagnosis}

When relaying only on clinical criteria, an acute onset of fever $\left(>38.5^{\circ} \mathrm{C}\right)$ accompanied with severe arthralgia or arthritis that cannot be explained by any other medical condition should be considered as a possible CHIKV case. When there is an epidemiological link (to reside, or visit an endemic area in the past 15 days), a suspected clinical case is determined as a probable CHIKV case. Since CHIKV geographical distribution overlaps with other pathologies with similar clinical manifestations, such as those causes by other arboviruses, a probable case can only become a confirmed case when it meets the necessary laboratory criteria [22].

Given the overlapping clinical spectrum of CHIKV, DENV, and ZIKV infections and their transmission by the same mosquito vectors, differential diagnosis is crucial for physicians to ensure good management and treatment for patients, especially when laboratory confirmation is not readily available for decision-making [6]. Retrospective studies of past CHIKV outbreaks have shown that together fever and arthralgia had a sensitivity between 73 and $84 \%$ with a positive predictive value around $75 \%$ and a negative predictive value between 44 and $84 \%$ [13, 41]. Furthermore, CHIKV-positive patients showed a greater incidence of skin rash, arthralgia on the feet and wrists, asthenia, higher temperature, and low frequency of digestive symptoms in comparison with CHIKV-negative patients [42].

When comparing CHIKV with DENV and ZIKV infections, there are main features that aid to perform a possible differential diagnosis, as shown in Table 1. The fever onset is more abrupt and its duration is much shorter in CHIKV infection in contrast with the gradual onset of DENV and ZIKV fever, where the latter courses in a lower grade $[43,44]$. Maculopapular rash is a skin manifestation reported in infections with these three arboviruses; nevertheless, it is more frequent in CHIKV infection [34]. During the early stages of the disease when skin rashes are absent, arthralgia can outstand CHIKV from other differential diagnoses $[45,46]$. Regarding hematological parameters, CHIKV 
infection is less prone to present thrombocytopenia and neutropenia when compared with DENV fever [47]. Moreover, studies have shown that a platelet count above $118 \times 10^{3} / \mathrm{ml}$ can function as a precise cutoff value to diagnose CHIKV with high accuracy and distinguish it from DENV infection [47]. Leukopenia is a common feature in the three infections, but it tends to be more pronounced in DENV disease, while CHIKV white blood cells counts normally range between $3,6-5 \times 10^{3} / \mathrm{ml}[47,48]$.

Despite the differences between each clinical presentation, this differential classification can only serve as a guide for the definition of suspected cases from one arboviral etiology over another. When patients lack "classical" signs or symptoms, none of the aforementioned causative agents should be ruled out without specific laboratory tests, especially since many atypical clinical presentations are observed.

\section{Clinical management and treatment of CHIKV infection}

Since an overlap in arboviral clinical presentation and vector distribution is present in many endemic areas, clinical identification of CHIKV must include further evaluation for DENV, ZIKV, and even Plasmodium sp. infection. When comparing severity during early stages of disease, DENV infection is more likely to quickly progress from a simple viral illness into a life-threatening condition than CHIKV or ZIKV infections. Therefore, it is prudent to assume dengue as the most important etiology in a differential diagnosis when the patient resides or comes from an endemic area. Dengue warning signs, such as persistent abdominal pain and vomiting, mucosal bleeding and thrombocytopenia, should always be monitored even if CHIKV or ZIKV infection are highly likely [49].

It is crucial to assess the presence of any underlying conditions or comorbidities such as diabetes, arterial hypertension, renal insufficiency, cardiomyopathies, and rheumatic illness, before management or treatment of patients, since CHIKV infection may cause decompensation of pre-existing diseases. Specific antiviral drugs, such as ribavirin, interferon alpha, chloroquine, arbidol, favipiravir, and furin inhibitors, have been recognized as effective against CHIKV in vitro and in vivo [50]. Nevertheless, none of them have been approved or licensed for CHIKV treatment in clinical settings. Therefore, treatment is focused on primarily supportive care, which includes the use of analgesic and anti-inflammatory medication, rehydration, and rest [27].

Non-steroidal anti-inflammatory drugs (NSAID) have been reported as the mainstay treatment for CHIKV infection but it should be completely avoided during the acute phase unless DENV has been confidently ruled out (by laboratory testing). It is well known that NSAIDs, especially aspirin, are contraindicated in DENV infection due to the increased risk of bleeding [51]. Corticosteroids should be avoided during the acute phase as well, since a strong rebound after treatment has been reported, and should only be used then during the sub-acute and chronic phases to treat moderate or severe joint pain. The most frequent used analgesics are paracetamol/acetaminophen and dipyrone in different dosages depending on pain severity [27]. Neuropathic involvement should be assessed and, if present, should be treated with anticonvulsants or antidepressants [52・].

Treatment during the chronic phase should consist in analgesics and NSAID if the patient reports mild to moderate pain. When pain is reported as severe or not responding to treatment, disease-modifying antirheumatic drugs 
(DMARDs), such as hydroxychloroquine and methotrexate, can be used [53]. Hydroxychloroquine is strongly suggested due to its anti-inflammatory effects on controlling joint pain, potential antiviral activity, and safety [53].

Since physical and daily activities tend to aggravate join inflammation and pain during the acute phase, absolute rest and hydration are strongly recommended [54]. Moreover, during the post-acute and chronic phase, persistent inflammation and pain may intensify patient suffering, thus psychological support is an essential component of the integrative approach for CHIKV patients [54].

During CHIKV acute phase, it is important to consistently inform patients about the potential complications and consequences of DENV, CHIKV, and ZIKV infections, especially if laboratory confirmation is not available or until a definitive diagnosis is determined. Furthermore, management and treatment of CHIKV illness should be addressed and discussed by a multidisciplinary group of health professionals in order to design a protocol that, while in agreement with the healthcare facility capacity, fulfills patients' potential needs.

Recommendations

- Even though CHIKV infection is rarely fatal, elderly, pediatric, and chronically ill patients should be carefully monitored for severe presentations.

- During the acute phase, CHIKV infection can be confused with other arboviroses, such as Dengue and Zika, so it is recommended to be aware of Dengue warning signs and avoid contraindicated medications for this disease.

- Despite the differences in clinical presentations of CHIKV, DENV, and ZIKV infections, laboratory tests are mandatory for confirmation.

- Rest, hydration, pharmacological treatment, and even psychological assessment should be part of the integrative support for CHIKV patients.

\section{Laboratory diagnosis of CHIKV infection}

\section{Detection of CHIKV}

Laboratory diagnosis becomes crucial in the identification of a confirmed CHIKV case and is the only option to confidently distinguish CHIKV from other possible etiologies. The main premise of the tests used in the laboratory is to detect CHIKV directly from samples by looking for components of the viral particle, such as nucleic acid and antigens, or to detect the specific host immune response triggered by CHIKV infection. Before choosing a diagnostic test, the physician should determine precisely the number of days since the onset of symptoms. This information, combined with the kinetics of viremia and antibody response, should be taken into account for test selection and interpretation. Factors such as purpose of testing and resources available are also important for this choice [51]. A combination of tests should be optimally employed for arriving at a CHIKV diagnosis, since each test possesses different sensitivity and specificity depending of the time passed after the onset of symptoms, as shown in Fig. 1.

After the onset of symptoms, patients become viremic during the next 5 days. This period is crucial for direct virus detection. Acute samples are more likely to result positive, thus a diagnostic test, when available, should be requested as soon as CHIKV is suspected. The tests that can be performed during this time are 


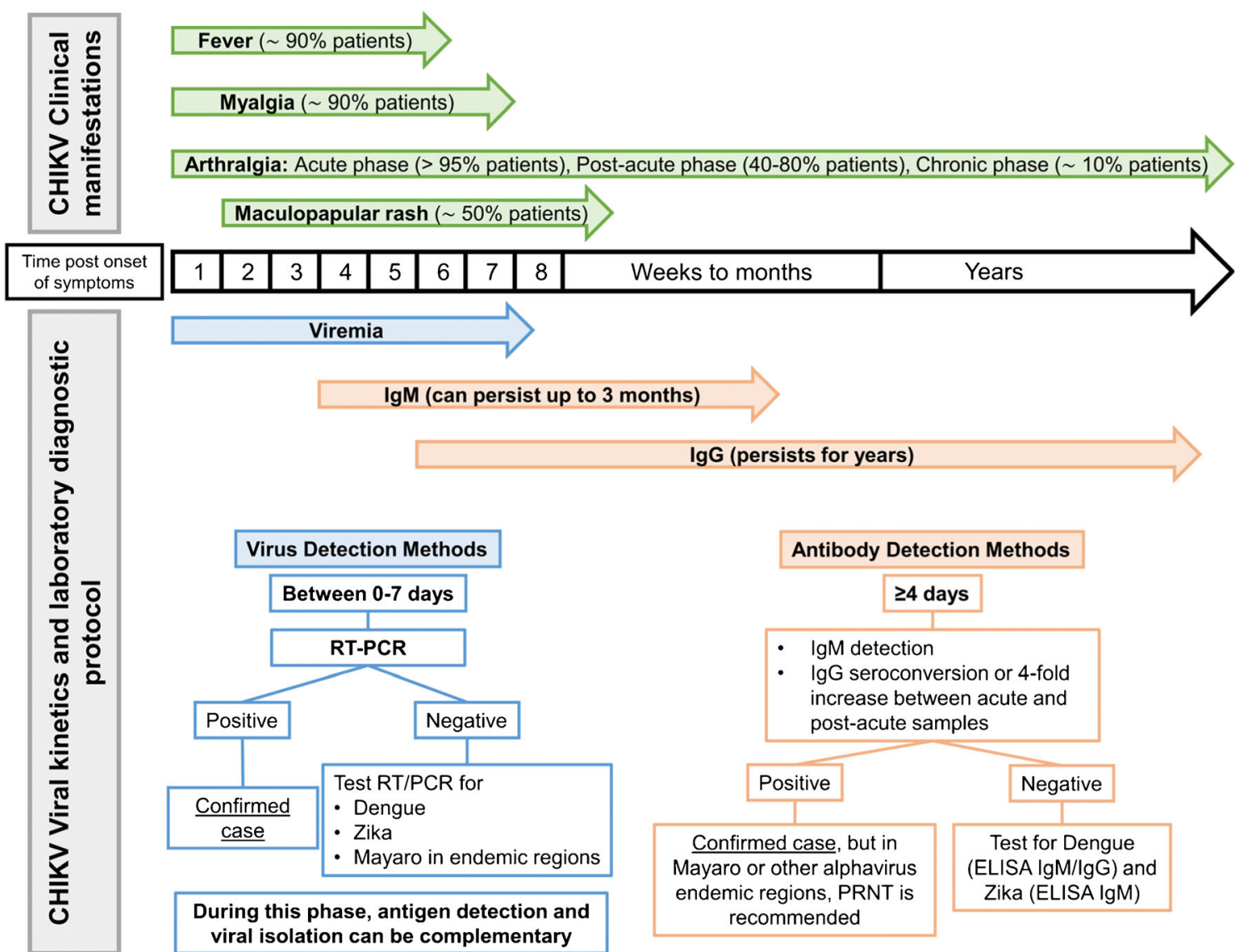

Fig. 1. CHIKV infection timeline regarding clinical manifestations, viral and antibody kinetics, and protocol for laboratory diagnosis

virus isolation (to be performed in high-containment laboratory facility, depending on the country's regulations) and detection of viral RNA or antigen [27]. Serum, plasma, whole blood, biopsies, and cerebrospinal fluid samples can be used for viral detection $[55,56]$.

As one of the important classically used diagnostic virological methods, virus isolation has the highest specificity and is usually defined as a gold standard for diagnosis. For CHIKV, virus isolation can be performed using mosquito cell lines, such as C6/36 (cloned from Ae. albopictus) and AP61 (clones from Ae. pseudoscutellaris), as well as mammalian cell lines such as VERO, LL-MCK, and BHK-21 [51]. In order to increase the possibility of virus isolation, at least two cell lines should be used, since replication in vitro can differ between genotypes and strains [57]. Even though virus isolation is considered a sensitive method, in practice, its sensitivity is highly variable [51]. Among the factors influencing the assay sensitivity are sample integrity, expertise of the laboratory staff, stability of the cell lines, and time after symptoms onset. Furthermore, the successful isolates should be characterized by in vitro methods, such as reverse-transcription polymerase chain reaction (RT-PCR) and immunofluorescence assays. In most countries, a biosafety level 3 laboratory is required for isolation, since most alphavirus can be transmitted by aerosols at high concentration [43]. Despite its advantages, viral isolation is not suitable for routine laboratory diagnostics, although it 
should be considered for surveillance purposes.

Nucleic acid and antigen detection approaches are usually more sensitive and have less turnaround time for a result when compared with virus isolation. Despite novel immunochromatographic assays to detect CHIKV antigens are being developed, these kits are not widely or commercially distributed and some of the available kits have dubious performance levels [58-60]. In the future, antigen detection methods should allow faster and cheaper diagnostic when compared with molecular approaches. Meanwhile, viral RNA detection is currently the preferred method for diagnosis since RNA can still be detected in blood samples a few days after viremia is over [51]. Several strategies for CHIKV nucleic acid detection have been developed but RT-PCR and real-time or quantitative PCR (qPCR) are the most widely used [61]. Another advantage of these methods is their versatility for making multiplex assays, allowing for simultaneous detection of more than one pathogen in the same run [62]. Nevertheless, multiplex assays could decrease sensitivity for each agent and primers designed for some CHIKV genotypes could exclude strains from other genotypes [63•]. CHIKV genotyping surveillance should be implemented in endemic regions in order to track changes that could modify the sensitivity of the current techniques. In some cases, to increase sensitivity of detection of emergent non-detectable strains and the emergence of other alphaviruses, genus-specific alphavirus RT-PCRs can be used $[64,65]$. These molecular assays must include the evaluation for DENV and $\mathrm{ZIKV}$, in order to make a more confident diagnosis of CHIKV by excluding other etiologies or detecting co-infections.

Recently, next-generation sequencing (NGS) technologies have been playing an important role in the clinical laboratory by modernizing how pathogens are detected $[66,67]$. NGS has the ability to provide broad detection of pathogens in a target-independent manner, improving the detection of all suspected, unsuspected or even novel arbovirus. Therefore, given the trend of emergence and re-emergence of novel arboviruses, NGS technologies should be considered as highly valuable tools for specialized diagnostics in arboviruses.

\section{Detection of the host immune response against CHIKV}

Once viremia declines, virus detecting techniques become less sensitive for diagnostic purposes. Therefore, detection of a specific CHIKV immune response, mainly antibodies, is the only strategy available.

Serological methods recurrently used are the enzyme-linked immunosorbent assay (ELISA), indirect immunofluorescence (IFA), hemagglutination inhibition (HI), neutralization, and immunochromatography test for rapid detection (RDT). The latter has shown to have the lowest performance level when compared with serological gold standards [68]. In order to extend the validity of serological tests, it is highly recommended to obtain a sample during the acute phase and another in the post-acute phase to establish seroconversion.

CHIKV infection is confirmed by serological criteria in only two scenarios: a positive result in specific IgM antibodies against CHIKV and when there is a fourfold increase in IgG titer in paired samples (seroconversion). CHIKVspecific IgM can be detected from 2 to 3 days after onset of symptoms and persist for weeks and even for 3 months [69]. On the other hand, IgG can be detected from day 4-6 and lasts for life [70]. Strikingly, these tests have shown cross-reaction with other alphavirus from the Semliki-Forest antigenic complex 
like Mayaro virus [71]. Consequently, it is important to address this potential cross-reaction by serological confirmation, with neutralization assays, when there is co-circulation of these viruses in the given geographical area. However, neutralization assays have the same demands as viral isolation with highly qualified personnel and laboratory facilities not available in most endemic regions.

Recommendations

- Determining the time after symptoms onset is crucial for choosing the appropriate diagnostic tests. Before day 8 post onset, virus detection tests can be used, but as time passes by, tests sensitivity decreases.

- Serological tests can be used from day 4 after onset for IgM detection. For diagnosis with only IgG detection, samples must be paired and seroconversion or fourfold increase in IgG titer must be shown. In regions with cocirculation of CHIKV and Mayaro virus, if confirmation depends solely on serological tests, neutralization assays may be needed.

- NGS technologies can improve arbovirus diagnostics and discovery of novel arboviruses after emergence and/or re-emergence.

\section{Conclusions}

As an emergent and re-emergent infection, the explosive nature of the last CHIKV epidemics has shown its increased adaptation to human hosts and their environments. This raises the concerns of augmented severity in newer outbreaks, as shown in the Americas. The overlapping clinical spectrum between $\mathrm{CHIKV}$, DENV, and ZIKV is the main challenge for the accurate clinical diagnosis and management of CHIKV infection. Multidisciplinary approaches regarding clinical, epidemiological and laboratory diagnosis of CHIKV infection and other circulating arboviruses should be put in place by health professionals in order to offer accurate management and treatment of this infection.

\section{Acknowledgements}

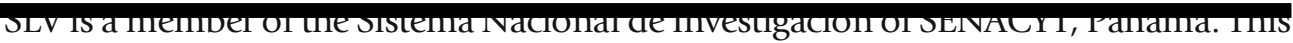
work was supported by Vicerrectoría de Investigación, Universidad de Costa Rica (803-B6-778) for the ARBORED.

\section{Compliance with ethical standards}

\section{Conflict of interest}

Claudio Soto-Garita declares that he has no conflict of interest. Jean-Paul Carrera declares that he has no conflict of interest.

Sandra López-Vergès declares that she has no conflict of interest.

Eugenia Corrales-Aguilar declares that she has no conflict of interest. 
Human and animal rights and informed consent

This article does not contain any studies with human or animal subjects performed by any of the authors.

\section{References and Recommended Reading}

Papers of particular interest, published recently, have been highlighted as:

- Of importance

$\bullet \quad$ Of major importance

1. Burt FJ, Rolph MS, Rulli NE, Mahalingam S, Heise MT. Chikungunya: a re-emerging virus. Lancet. 2012;379(9816):662-71.

2. Mason PJ, Haddow AJ. An epidemic of virus disease in Southern Province, Tanganyika Territory, in 19521953. Trans R Soc Trop Med Hyg. 1957;51:238-40.

3. Lumsden WH. An epidemic of virus disease in Southern Province, Tanganyika Territory, in 1952-53. II. General description and epidemiology. Trans R Soc Trop Med Hyg. 1955;49:33-57.

4. Morrison TE. Reemergence of chikungunya virus. J Virol. 2014;88:11644-7.

5. Weaver $\mathrm{SC}$, Lecuit $\mathrm{M}$. Chikungunya virus and the global spread of a mosquito-borne disease. N Engl J Med. 2015;372:1231-9.

6. Thiberville SD, Moyen N, Dupuis-Maguiraga L, Nougairede A, Gould EA, Roques P, et al. Chikungunya fever: epidemiology, clinical syndrome, pathogenesis and therapy. Antiviral Res. 2013;99(3):345-70.

7. $\quad$ Weaver SC, Forrester NL. Chikungunya: evolutionary history and recent epidemic spread. Antiviral Res. 2015;120:32-9.

This review explores the emergence and re-emergence of CHIKV and its evolutionary history, and provides a detailed description of the introduction to the Americas.

8. Halstead SB. Reappearance of chikungunya, formerly called dengue, in the Americas. Emerg Infect Dis. 2015;21:557-61.

9. Kuno G. A re-examination of the history of etiologic confusion between dengue and chikungunya. PLoS Negl Trop Dis. 2015;9(11):e0004101.

10. Petersen LR, Powers AM. Chikungunya: epidemiology. F1000Research. 2016;5:82.

11. Sergon K, Yahaya AA, Brown J, Bedja SA, Mlindasse M, Agata N, et al. Seroprevalence of chikungunya virus infection on Grande Comore Island, Union of the Comoros, 2005. Am J Trop Med Hyg. 2007;76:118993.

12. Pialoux G, Gaüzère BA, Jauréguiberry S, Strobel M. Chikungunya, an epidemic arbovirosis. Lancet Infect Dis. 2007;7(5):319-27.

13. Renault P, Solet JL, Sissoko D, Balleydier E, Larrieu S, Filleul L, et al. A major epidemic of chikungunya virus infection on Réunion Island, France, 2005-2006. Am J Trop Med Hyg. 2007;77:727-31.

14.• Burt FJ, Chen W, Miner JJ, Lenschow DJ, Merits A, Schnettler E, et al. Chikungunya virus: an update on the biology and pathogenesis of this emerging pathogen. Lancet Infect Dis. 2017;17(4):e107-17.

This paper offers a deep insight in CHIKV pathogenesis and the current advances in CHIKV animal models.

15. Schuffenecker I, Iteman I, Michault A, Murri S, Frangeul $\mathrm{L}$, Vaney MC, et al. Genome microevolution of chikungunya viruses causing the Indian Ocean outbreak. PLoS Med. 2006;3:1058-70.

16. Bonilauri $\mathrm{P}$, Bellini R, Calzolari M, Angelini R, Venturi L, Fallacara F, et al. Chikungunya virus in Aedes albopictus, Italy. Emerg Infect Dis. 2008;14(5):852-4.

17. Leparc-Goffart I, Nougairede A, Cassadou S, Prat C, De Lamballerie X. Chikungunya in the Americas. Lancet. 2014;383(9916):514.

18. Yactayo S, Staples JE, Millot V, Cibrelus L, RamonPardo P. Epidemiology of chikungunya in the americas. J Infect Dis. 2016;214(Suppl 5):S441-5.

19. Da Costa AC, Thézé J, Komninakis SCV, Sanz-Duro RL, Felinto MRL, Moura LCC, et al. Spread of chikungunya virus east/central/South African genotype in northeast Brazil. Emerg Infect Dis. 2017;23(10):1742-4.

20. Sahadeo NSD, Allicock OM, De Salazar PM, Auguste AJ, Widen S, Olowokure B, et al. Understanding the evolution and spread of chikungunya virus in the Americas using complete genome sequences. Virus Evol. 2017;3(1):vex010. Available from: https:// academic.oup.com/ve/article-lookup/doi/10.1093/ve/ vex010

21. Schilte C, Staikovsky F, Couderc T, Madec Y, Carpentier F, Kassab S, et al. Chikungunya virus-associated longterm arthralgia: a 36-month prospective longitudinal study. PLoS Negl Trop Dis. 2013;7(3):e2137.

22. OMS. OMS | Chikungunya. WHO. 2016.

23. Sissoko D, Malvy D, Ezzedine K, Renault P, Moscetti F, Ledrans $\mathrm{M}$, et al. Post-epidemic chikungunya disease on reunion island: course of rheumatic manifestations and associated factors over a 15-month period. PLoS Negl Trop Dis. 2009;3(3):e389.

24. Sissoko D, Malvy D, Giry C, Delmas G, Paquet C, Gabrie P, et al. Outbreak of chikungunya fever in Mayotte, Comoros archipelago, 2005-2006. Trans R Soc Trop Med Hyg. 2008;102:780-6.

25. Yoon IK, Alera MT, Lago CB, Tac-An IA, Villa D, Fernandez S, et al. High Rate of subclinical chikungunya virus infection and association of neutralizing antibody with protection in a prospective cohort in the Philippines. PLoS Negl Trop Dis. 2015;9(5):e0003764. 
26. Kuan G, Ramirez S, Gresh L, Ojeda S, Melendez M, Sanchez N, et al. Seroprevalence of anti-chikungunya virus antibodies in children and adults in Managua, Nicaragua, after the first chikungunya epidemic, 20142015. PLoS Negl Trop Dis. 2016;10(6):e0004773.

27. Da Cunha RV, Trinta KS. Chikungunya virus: clinical aspects and treatment-a review. Mem Inst Oswaldo Cruz. 2017;112:523-31.

28. Queyriaux B, Simon F, Grandadam M, Michel R, Tolou $\mathrm{H}$, Boutin JP. Clinical burden of chikungunya virus infection. Lancet Infect Dis. 2008;8(1):2-3.

29. Lam SK, Chua KB, Hooi PS, Rahimah MA, Kumari S, Tharmaratnam $\mathrm{M}$, et al. Chikungunya infection-an emerging disease in Malaysia. Southeast Asian J Trop Med Public Health. 2001;32:447-51.

30. Rezza G, Nicoletti L, Angelini R, Romi R, Finarelli A, Panning $M$, et al. Infection with chikungunya virus in Italy: an outbreak in a temperate region. Lancet. 2007;370:1840-6.

31. Borgherini G, Poubeau P, Staikowsky F, Lory M, Moullec NL, Becquart JP, et al. Outbreak of chikungunya on Reunion Island: early clinical and laboratory features in 157 adult patients. Clin Infect Dis. 2007;44:1401-7.

32. Seetharam KA, Sridevi K, Vidyasagar P. Cutaneous manifestations of chikungunya fever. Indian Pediatr. 2012;49:51-3.

33. Ritz N, Hufnagel M, Gérardin P. Chikungunya in children. Pediatr Infect Dis J. 2015;34:789-91.

34. Busch M, Erickson G. An overview of Chikungunya virus. J Am Acad Physician Assist. 2015;28:54-7.

35. Farnon EC, Sejvar JJ, Staples JE. Severe disease manifestations associated with acute chikungunya virus infection. Crit Care Med. 2008;36:2682-3.

36. Bandeira AC, Campos GS, Sardi SI, Rocha VFD, Rocha GCM. Neonatal encephalitis due to chikungunya vertical transmission: First report in Brazil. IDCases. 2016;5:57-9.

37. Rajapakse S, Rodrigo C, Rajapakse A. Atypical manifestations of chikungunya infection. Trans R Soc Trop Med Hyg. 2010;104(2):89-96.

38. Chopra A, Anuradha V, Ghorpade R, Saluja M. Acute chikungunya and persistent musculoskeletal pain following the 2006 Indian epidemic: a 2-year prospective rural community study. Epidemiol Infect. 2012;140:842-50.

39. Waymouth HE, Zoutman DE, Towheed TE. Chikungunya-related arthritis: case report and review of the literature. Semin Arthritis Rheum. 2013;43:2738.

40. Dupuis-Maguiraga L, Noret M, Brun S, Le Grand R, Gras $G$, Roques P. Chikungunya disease: infectionassociated markers from the acute to the chronic phase of arbovirus-induced arthralgia. PLoS Negl Trop Dis. 2012;6(3):e1446.

41. Nkoghe D, Kassa RF, Caron M, Grard G, Mombo I, Bikié B, et al. Clinical forms of chikungunya in Gabon, 2010. PLoS Negl Trop Dis. 2012;6(2):e1517.
42. Staikowsky F, Talarmin F, Grivard P, Souab A, Schuffenecker I, Le Roux K, et al. Prospective study of chikungunya virus acute infection in the Island of $\mathrm{La}$ Réunion during the 2005-2006 outbreak. PLoS One. 2009;4(10):e7603.

43. Word Health Organization. Guidelines on clinical management of chikungunya fever. Geneva: World Heal. Organ.; 2008. p. 18.

44. Ioos S, Mallet HP, Leparc Goffart I, Gauthier V, Cardoso T, Herida M. Current zika virus epidemiology and recent epidemics. Med Mal Infect. 2014;44(7):302-7.

45. Laoprasopwattana K, Kaewjungwad L, Jarumanokul R, Geater A. Differential diagnosis of chikungunya, dengue viral infection and other acute febrile illnesses in children. Pediatr Infect Dis J. 2012;31:459-63.

46. Taraphdar D, Sarkar A, Mukhopadhyay BB, Chatterjee S. A comparative study of clinical features between monotypic and dual infection cases with chikungunya virus and dengue virus in West Bengal, India. Am J Trop Med Hyg. 2012;86:720-3.

47. Lee VJ, Chow A, Zheng X, Carrasco LR, Cook AR, Lye DC, et al. Simple clinical and laboratory predictors of chikungunya versus dengue infections in adults. PLoS Negl Trop Dis. 2012;6(9):e1786.

48. Chen LH, Wilson ME. Dengue and chikungunya infections in travelers. Curr Opin Infect Dis. 2010;23(5):438-44.

49. Patterson J, Sammon M, Garg M. Dengue, zika and chikungunya: emerging arboviruses in the New World. West J Emerg Med. 2016;17:671-9.

50. Parashar D, Cherian S. Antiviral perspectives for chikungunya virus. Biomed Res Int. 2014;2014:631642.

51. Mardekian SK, Roberts AL. Diagnostic options and challenges for dengue and chikungunya viruses. Biomed Res Int. 2015;2015:834371.

52. - de Brito CAA, von Sohsten AKA, de Sá Leitão CC, de Brito RCCM, De Azevedo Valadares LD, da Fonte CAM, et al. Pharmacologic management of pain in patients with chikungunya: a guideline. Rev Soc Bras Med Trop. 2016;49(6):668-79.

This review proposes a complete guideline for pharmacological treatment of CHIKV infection, based on clinical evidence in an endemic region.

53. Delogu I, De Lamballerie X. Chikungunya disease and chloroquine treatment. J Med Virol. 2011;83(6):1058-9.

54. Javelle E, Ribera A, Degasne I, Gaüzère BA, Marimoutou C, Simon F. Specific management of postchikungunya rheumatic disorders: a retrospective study of 159 cases in Reunion Island from 2006-2012. PLoS Negl Trop Dis. 2015;9(3):e0003603.

55. Mishra B, Sharma M, Pujhari SK, Ratho RK, Gopal DVRS, Kumar CN, et al. Utility of multiplex reverse transcriptase-polymerase chain reaction for diagnosis and serotypic characterization of dengue and chikungunya viruses in clinical samples. Diagn Microbiol Infect Dis. 2011;71:118-25. 
56. Puttamallesh VN, Sreenivasamurthy SK, Singh PK, Harsha HC, Ganjiwale A, Broor S, et al. Proteomic profiling of serum samples from chikungunya-infected patients provides insights into host response. Clin Proteomics. 2013;10(1):14.

57. Wikan N, Sakoonwatanyoo P, Ubol S, Yoksan S, Smith DR. Chikungunya virus infection of cell lines: analysis of the east, central and south African lineage. PLoS One. 2012;7(1):e31102.

58. Kashyap RS, Morey SH, Ramteke SS, Chandak NH, Parida M, Deshpande PS, et al. Diagnosis of chikungunya fever in an Indian population by an indirect enzyme-linked immunosorbent assay protocol based on an antigen detection assay: a prospective cohort study. Clin Vaccine Immunol. 2010;17:291-7.

59. Shukla J, Khan M, Tiwari M, Sannarangaiah S, Sharma $\mathrm{S}$, Rao PVL, et al. Development and evaluation of antigen capture ELISA for early clinical diagnosis of chikungunya. Diagn Microbiol Infect Dis. 2009;65:142-9.

60. Okabayashi T, Sasaki T, Masrinoul P, Chantawat N, Yoksan S, Nitatpattana N, et al. Detection of chikungunya virus antigen by a novel rapid immunochromatographic test. J Clin Microbiol. 2015;53:382-8.

61. Johnson BW, Russell BJ, Goodman CH. Laboratory diagnosis of chikungunya virus infections and commercial sources for diagnostic assays. J Infect Dis. 2016;14(suppl 5):S471-4.

62. Simmons M, Myers T, Guevara C, Jungkind D, Williams M, Houng HS. Development and validation of a quantitative, one-step, multiplex, real-time reverse transcriptase PCR assay for detection of dengue and chikungunya viruses. J Clin Microbiol. 2016;54:1766-73.

63. Duncan R, Kourout M, Grigorenko E, Fisher C, Dong $\mathrm{M}$. Advances in multiplex nucleic acid diagnostics for blood-borne pathogens: Promises and pitfalls. Expert Rev Mol Diagn. 2016;16(1):83-95.

This paper provides an exhausting review of the advantages and disadvantages of multiplex assays, which are used for arbovirus diagnostics.

64. Sánchez-Seco MP, Rosario D, Quiroz E, Guzmán G, Tenorio A. A generic nested-RT-PCR followed by sequencing for detection and identification of members of the alphavirus genus. J Virol Methods. 2001;95:153-61.

65. Giry C, Roquebert B, Li-Pat-Yuen G, Gasque P, JaffarBandjee MC. Simultaneous detection of chikungunya virus, dengue virus and human pathogenic Leptospira genomes using a multiplex TaqMan ${ }^{\circledR}$ assay. BMC Microbiol. 2017;17(1):105.

66. Capobianchi MR, Giombini E, Rozera G. Nextgeneration sequencing technology in clinical virology. Clin Microbiol Infect. 2013;19(1):15-22.

67. Datta S. Next-generation sequencing in clinical virology: discovery of new viruses. World J Virol. 2015;4:265.

68. Prat CM, Flusin O, Panella A, Tenebray B, Lanciotti R, Leparc-Goffart I. Evaluation of commercially available serologic diagnostic tests for chikungunya virus. Emerg. Infect. Dis. 2014;20:2129-32.

69. Reddy V, Ravi V, Desai A, Parida M, Powers AM, Johnson BW. Utility of IgM ELISA, TaqMan real-time PCR, reverse transcription PCR, and RT-LAMP assay for the diagnosis of chikungunya fever. J Med Virol. 2012;84:1771-8.

70. Yap G, Pok KY, Lai YL, Hapuarachchi HC, Chow A, Leo YS, et al. Evaluation of chikungunya diagnostic assays: differences in sensitivity of serology assays in two independent outbreaks. PLoS Negl Trop Dis. 2010;4:e753.

71. Hassing RJ, Leparc-Goffart I, Tolou H, van Doornum G, van Genderen PJ. Cross-reactivity of antibodies to viruses belonging to the Semliki forest serocomplex. Euro Surveill. 2010;15(23):pii=19588. 